This item was submitted to Loughborough's Research Repository by the author.

Items in Figshare are protected by copyright, with all rights reserved, unless otherwise indicated.

Nonexpanding impulsive gravitational waves with an arbitrary cosmological constant

PLEASE CITE THE PUBLISHED VERSION

LICENCE

CC BY-NC-ND 4.0

REPOSITORY RECORD

Podolsky, J., and J.B. Griffiths. 2019. "Nonexpanding Impulsive Gravitational Waves with an Arbitrary Cosmological Constant”. figshare. https://hdl.handle.net/2134/834. 


\title{
Nonexpanding impulsive gravitational waves with an arbitrary cosmological constant
}

\author{
J. Podolský* \\ Department of Theoretical Physics, Charles University, \\ V Holešovičkách 2, 18000 Prague 8, Czech Republic. \\ and J. B. Griffiths ${ }^{\dagger}$ \\ Department of Mathematical Sciences, Loughborough University \\ Loughborough, Leics. LE11 3TU, U.K.
}

August 2, 1999

\begin{abstract}
Exact solutions for nonexpanding impulsive waves in a background with nonzero cosmological constant are constructed using a "cut and paste" method. These solutions are presented using a unified approach which covers the cases of de Sitter, anti-de Sitter and Minkowski backgrounds. The metrics are presented in continuous and distributional forms, both of which are conformal to the corresponding metrics for impulsive $p p$-waves, and for which the limit as $\Lambda \rightarrow 0$ can be made explicitly.
\end{abstract}

PACS: 04.20.Jb; 04.30.Nk

Keywords: Impulsive gravitational waves, (anti-)de Sitter space.

Penrose [1] has presented a geometrical method for the construction of plane (nonexpanding) and spherical (expanding) impulsive gravitational waves in a Minkowski background by cutting the space-time along a null hypersurface and then re-attaching the two pieces with a suitable warp. The first case leads to impulsive $p p$-waves. The second case describing expanding impulsive waves [2]-[4] has been extended to backgrounds with a nonzero cosmological constant $\Lambda$ [5]. However, the method has not previously been explicitly used for the construction of nonexpanding waves in backgrounds with $\Lambda \neq 0$, although such solutions are already known [6]-[8]. The purpose of the present letter is to derive these solutions using the Penrose method. Remarkably, this approach leads to a convenient unified representation of the whole family of solutions in which it is possible to set $\Lambda=0$ explicitly.

\footnotetext{
${ }^{*}$ E-mail: Podolsky@mbox.troja.mff.cuni.cz

${ }^{\dagger} \mathrm{E}-\mathrm{mail}$ : J.B.Griffiths@Lboro.ac.uk
} 
Let us first recall the line element for a space-time of constant curvature in the manifestly conformally flat form

$$
\mathrm{d} s_{0}^{2}=\frac{2 \mathrm{~d} u \mathrm{~d} v-2 \mathrm{~d} \zeta \mathrm{d} \bar{\zeta}}{\left[1-\frac{1}{6} \Lambda(u v-\zeta \bar{\zeta})\right]^{2}}
$$

where $\Lambda$ is the cosmological constant. This is de Sitter space when $\Lambda>0$, anti-de Sitter space when $\Lambda<0$ and Minkowski space when $\Lambda=0$. As is well known, the (anti-)de Sitter space-time can be represented as the 4-dimensional hyperboloid

$$
Z_{0}^{2}-Z_{1}^{2}-Z_{2}^{2}-Z_{3}^{2}-\epsilon Z_{4}^{2}=-\epsilon a^{2}, \quad a^{2}=\frac{3}{\epsilon \Lambda},
$$

in the flat 5-dimensional space $\mathrm{d} s_{0}^{2}=\mathrm{d} Z_{0}{ }^{2}-\mathrm{d} Z_{1}{ }^{2}-\mathrm{d} Z_{2}{ }^{2}-\mathrm{d} Z_{3}{ }^{2}-\epsilon \mathrm{d} Z_{4}{ }^{2}$, where $\epsilon=1$ for $\Lambda>0$ and $\epsilon=-1$ for $\Lambda<0$. The coordinates of the metric (1) form a suitable parameterization of the hyperboloid in which

$$
\begin{aligned}
Z_{0} & =\frac{1}{\sqrt{2}}(u+v)\left[1-\frac{1}{6} \Lambda(u v-\zeta \bar{\zeta})\right]^{-1}, \\
Z_{1} & =\frac{1}{\sqrt{2}}(u-v)\left[1-\frac{1}{6} \Lambda(u v-\zeta \bar{\zeta})\right]^{-1}, \\
Z_{2} & =\frac{1}{\sqrt{2}}(\zeta+\bar{\zeta})\left[1-\frac{1}{6} \Lambda(u v-\zeta \bar{\zeta})\right]^{-1}, \\
Z_{3} & =-i \frac{1}{\sqrt{2}}(\zeta-\bar{\zeta})\left[1-\frac{1}{6} \Lambda(u v-\zeta \bar{\zeta})\right]^{-1}, \\
Z_{4} & =\sqrt{\frac{3}{|\Lambda|}}\left[\frac{1+\frac{1}{6} \Lambda(u v-\zeta \bar{\zeta})}{1-\frac{1}{6} \Lambda(u v-\zeta \bar{\zeta})}\right] .
\end{aligned}
$$

Inversely, this is given by $u=\frac{1}{\sqrt{2}}\left(Y_{0}+Y_{1}\right), v=\frac{1}{\sqrt{2}}\left(Y_{0}-Y_{1}\right), \zeta=\frac{1}{\sqrt{2}}\left(Y_{2}+i Y_{3}\right)$ where $Y_{\alpha}=2 a Z_{\alpha} /\left(Z_{4}+a\right)$ with $\alpha=0,1,2,3$. It may be observed that these coordinates cover the complete hyperboloid, although there is a coordinate singularity along the section $Z_{4}=-a$.

Let us now consider the transformation $u=U, v=V+H+U H_{Z} H_{\bar{Z}}, \zeta=Z+U H_{\bar{Z}}$ applied to the line element $(1)$, where $H=H(Z, \bar{Z})$ is an arbitrary real function. This results in the metric

$$
\mathrm{d} s_{0}^{2}=\frac{2 \mathrm{~d} U \mathrm{~d} V-2\left|\mathrm{~d} Z+U\left(H_{Z \bar{Z}} \mathrm{~d} Z+H_{\bar{Z} \bar{Z}} \mathrm{~d} \bar{Z}\right)\right|^{2}}{\left[1-\frac{1}{6} \Lambda(U V-Z \bar{Z}+U G)\right]^{2}},
$$

where $G=H-Z H_{Z}-\bar{Z} H_{\bar{Z}}$.

Following Penrose's "cut and paste" method [1], we may now take the line element (1) with $u=U, v=V$ and $\zeta=Z$ for $U<0$ and combine this with (3) for $U>0$. The resulting line element

$$
\mathrm{d} s^{2}=\frac{2 \mathrm{~d} U \mathrm{~d} V-2\left|\mathrm{~d} Z+U \Theta(U)\left(H_{Z \bar{Z}} \mathrm{~d} Z+H_{\bar{Z} \bar{Z}} \mathrm{~d} \bar{Z}\right)\right|^{2}}{\left[1-\frac{1}{6} \Lambda(U V-Z \bar{Z}+U \Theta(U) G)\right]^{2}},
$$

where $\Theta(U)$ is the Heaviside step function, is continuous across the null hypersurface $U=0=u$. However, the discontinuity in the derivatives of the metric yields impulsive components in the curvature tensor proportional to the Dirac $\delta$-function. These are interpreted as impulsive waves in de Sitter, anti-de Sitter or Minkowski backgrounds. For $\Lambda=0$, this reduces to the well known Rosen form for impulsive $p p$-waves [9], [10]. 
We may observe from (1) that the geometry of the wavefront $u=0$ is described by the 2-metric

$$
\mathrm{d} \sigma^{2}=-\frac{2 \mathrm{~d} \zeta \mathrm{d} \bar{\zeta}}{\left[1+\frac{1}{6} \Lambda \zeta \bar{\zeta}\right]^{2}}
$$

which is a 2-dimensional space of constant gaussian curvature $K=\Lambda / 3$. When $\Lambda=0$ the impulsive wave surface is a plane, for $\Lambda>0$ it is a sphere, while for $\Lambda<0$ it is a hyperboloid. For $\Lambda \neq 0$, the geometry of these surfaces has been described in detail in [11].

Now, the explicit form of the complete transformation formed by combining those above is given by

$$
\begin{aligned}
u & =U \\
v & =V+H \Theta(U)+U \Theta(U) H_{Z} H_{\bar{Z}} \\
\zeta & =Z+U \Theta(U) H_{\bar{Z}} .
\end{aligned}
$$

This is discontinuous at $u=0$ in such a way that

$$
(u=0, v, \zeta, \bar{\zeta})_{M^{-}}=(u=0, v-H(\zeta, \bar{\zeta}), \zeta, \bar{\zeta})_{M^{+}}
$$

which is exactly the Penrose junction condition for reattaching the two halves of the space-time $M^{-}(u<0)$ and $M^{+}(u>0)$ with a "warp".

Significantly, the transformation (6), taking into account the terms which arise from the derivatives of $\Theta(u)$, relates the continuous form of the impulsive wave metric (4), not to the initial metric (1), but to the following metric which also includes an impulsive component explicitly located on the wavefront $u=0$

$$
\mathrm{d} s^{2}=\frac{2 \mathrm{~d} u \mathrm{~d} v-2 \mathrm{~d} \zeta \mathrm{d} \bar{\zeta}-2 H(\zeta, \bar{\zeta}) \delta(u) \mathrm{d} u^{2}}{\left[1-\frac{1}{6} \Lambda(u v-\zeta \bar{\zeta})\right]^{2}} .
$$

This represents a nonexpanding impulsive wave in any background space-time of constant curvature.

The above result is well known for impulsive waves in a Minkowski background, where it is exactly the standard Brinkmann form for a general impulsive $p p$-wave. However, the above form has not previously been given explicitly for the case when $\Lambda \neq 0$. For $\Lambda<0$, an equivalent form (in $d$-dimensions) has been used in [12].

It may be observed that the metric (7) is conformal to the general impulsive $p p$-wave. In this context, we recall that Siklos [13] has proved that Einstein spaces conformal to $p p$-waves only occur when $\Lambda<0$. However, it would appear that the impulsive case is a counter-example to this result.

In fact, the metric (7) is a suitable parameterisation of a class of nonexpanding impulsive wave solutions described in a 5-dimensional formalism in [7]:

$$
\mathrm{d} s^{2}=\mathrm{d} Z_{0}^{2}-\mathrm{d} Z_{1}^{2}-\mathrm{d} Z_{2}^{2}-\mathrm{d} Z_{3}^{2}-\epsilon \mathrm{d} Z_{4}^{2}-\tilde{H}\left(Z_{2}, Z_{3}, Z_{4}\right) \delta\left(Z_{0}+Z_{1}\right)\left(\mathrm{d} Z_{0}+\mathrm{d} Z_{1}\right)^{2} .
$$

Indeed, using (2), we obtain exactly (7) where

$$
H(\zeta, \bar{\zeta})=\frac{1}{\sqrt{2}}\left(1+\frac{1}{6} \Lambda \zeta \bar{\zeta}\right) \tilde{H}(\zeta, \bar{\zeta})
$$

In this relation, the parameterisation (2), restricted to the impulsive wave surface $u=0$, is used to express the arguments of $\tilde{H}$ in terms of $\zeta$ and $\bar{\zeta}$ only. 
The above solutions can describe impulsive gravitational waves or impulses of null matter. Using the tetrad frame $\ell^{\mu}=\Omega \delta_{2}^{\mu}, m^{\mu}=\Omega \frac{1}{\sqrt{2}}\left(\delta_{3}^{\mu}+i \delta_{4}^{\mu}\right), n^{\mu}=\Omega\left(\delta_{1}^{\mu}+H \delta(u) \delta_{2}^{\mu}\right)$ where $\Omega=1-\frac{1}{6} \Lambda(u v-\zeta \bar{\zeta})$, the nonzero components of the Weyl and Ricci tensors are

$$
\begin{aligned}
\Psi_{4} & =-\left(1+\frac{1}{6} \Lambda \zeta \bar{\zeta}\right)^{2} H_{\zeta \zeta} \delta(u), \\
\Phi_{22} & =-\left(1+\frac{1}{6} \Lambda \zeta \bar{\zeta}\right)\left[\left(1+\frac{1}{6} \Lambda \zeta \bar{\zeta}\right) H_{\zeta \bar{\zeta}}+\frac{1}{6} \Lambda\left(H-\zeta H_{\zeta}-\bar{\zeta} H_{\bar{\zeta}}\right)\right] \delta(u) .
\end{aligned}
$$

With (8) the vacuum field equations $\Phi_{22}=0$ can then be expressed as

$$
\left(1+\frac{1}{6} \Lambda \zeta \bar{\zeta}\right)^{2} \tilde{H}_{\zeta \bar{\zeta}}+\frac{1}{3} \Lambda \tilde{H}=0
$$

which is simply $\left(\Delta+\frac{2}{3} \Lambda\right) \tilde{H}=0$, where $\Delta$ is the Laplacian operator on a 2-dimensional impulsive wave surface (5). This generalises the well known vacuum field equations for impulsive waves in a Minkowski background. A general solution of this equation (9) is

$$
\tilde{H}(\zeta, \bar{\zeta})=\left(f_{\zeta}+\bar{f}_{\bar{\zeta}}\right)-\frac{\Lambda}{3} \frac{\bar{\zeta} f+\zeta \bar{f}}{\left(1+\frac{1}{6} \Lambda \zeta \bar{\zeta}\right)}
$$

where $f(\zeta)$ is an arbitrary function of $\zeta$. Thus, the general vacuum solution for the metric (7) is given by $\sqrt{2} H(\zeta, \bar{\zeta})=\left(1+\frac{1}{6} \Lambda \zeta \bar{\zeta}\right)\left(f_{\zeta}+\bar{f}_{\bar{\zeta}}\right)-\frac{1}{3} \Lambda(\bar{\zeta} f+\zeta \bar{f})$. This describes an impulsive gravitational wave in which $\Psi_{4}=-\frac{1}{\sqrt{2}}\left(1+\frac{1}{6} \Lambda \zeta \bar{\zeta}\right)^{3} f_{\zeta \zeta \zeta} \delta(u)$. The space-time is conformally flat everywhere when the function $f$ is at most quadratic in $\zeta$. However, solutions of (9) necessarily contain singularities. These are located on the wavefronts and may be considered as null sources of the impulsive gravitational waves.

For example, the Aichelburg-Sexl solution [14] obtained by boosting the Schwarzschild metric is given by $f_{0}=\frac{1}{2} \zeta(\log \zeta-1)$. Similarly, the Hotta-Tanaka solution [6] obtained by boosting the Schwarzschild-(anti-)de Sitter metric is given by $f_{0}=\frac{1}{2} \zeta\left(\log \zeta+\frac{1}{2} \log \frac{1}{6}|\Lambda|\right)$. For these

$$
\begin{array}{ll}
\Lambda=0: & \sqrt{2} H_{0}=\frac{1}{2} \log \zeta \bar{\zeta}, \\
\Lambda \neq 0: & \sqrt{2} H_{0}=\frac{1}{2}\left(1-\frac{1}{6} \Lambda \zeta \bar{\zeta}\right) \log \left(\frac{1}{6}|\Lambda| \zeta \bar{\zeta}\right)+\left(1+\frac{1}{6} \Lambda \zeta \bar{\zeta}\right) .
\end{array}
$$

In the second expression, the limit as $\Lambda \rightarrow 0$ differs from the first only by a constant term that can be transformed away. These solutions describe gravitational waves generated by null monopole point particles in backgrounds of constant curvature. Further explicit solutions representing impulsive gravitational waves generated by null particles with arbitrary multipole structure in these backgrounds have been given previously in [15] and [7]. For dipole and quadrupole sources, the solutions are given by $f_{1}=\left(1-\frac{1}{6} \Lambda \zeta^{2}\right) \log \zeta+\log \frac{1}{6}|\Lambda|$ and $f_{2}=-\zeta^{-1}+\frac{1}{36} \Lambda^{2} \zeta^{3}$, for which

$$
\begin{aligned}
\sqrt{2} H_{1} & =\left(\frac{1}{\zeta}+\frac{1}{\bar{\zeta}}\right)\left(1-\left[\frac{1}{6} \Lambda \zeta \bar{\zeta}\right]^{2}\right)-\frac{1}{3} \Lambda(\zeta+\bar{\zeta}) \log \left(\frac{1}{6}|\Lambda| \zeta \bar{\zeta}\right), \\
\sqrt{2} H_{2} & =\left(\frac{1}{\zeta^{2}}+\frac{1}{\bar{\zeta}^{2}}\right)\left(1+\frac{1}{6} \Lambda \zeta \bar{\zeta}\right)^{3},
\end{aligned}
$$

respectively. It may be noted that, for these and higher multipole terms, the limit for the metric components as $\Lambda \rightarrow 0$ can be performed explicitly. This further demonstrates the suitability of these coordinates for discussing the above class of impulsive solutions in a unified way. 


\section{Acknowledgments}

This work was supported by a visiting fellowship from the Royal Society and, in part, by the grant GACR-202/99/0261 of the Czech Republic.

\section{References}

[1] R. Penrose, General Relativity ed L O'Raifeartaigh (Clarendon, Oxford, 1972) 101

[2] R. Gleiser and J. Pullin, Class. Quantum Grav. 6 (1989) L141

[3] Y. Nutku and R Penrose, Twistor Newsletter No. 34, 11 May (1992) 9

[4] P. A. Hogan, Phys. Rev. Lett. 70 (1993) 117

[5] P. A. Hogan, Phys. Lett. A 171 (1992) 21

[6] M. Hotta and T. Tanaka, Class. Quantum Grav. 10 (1993) 307

[7] J. Podolský and J. B. Griffiths, Class. Quantum Grav. 15 (1998) 453

[8] J. Podolský, Class. Quantum Grav. 15 (1998) 3229

[9] J. Podolský and K. Veselý, Phys. Lett. A 241 (1998) 145

[10] M. Kunzinger and R. Steinbauer, Class. Quantum Grav. 16 (1999) 1255

[11] J. Podolský and J. B. Griffiths, Phys. Rev. D 56 (1997) 4756

[12] G. T. Horowitz and N. Itzhaki, JHEP 9902 (1999) 010

[13] S. T. C. Siklos, Galaxies, axisymmetric systems and relativity ed M A H MacCallum (Cambridge, 1985) 247

[14] P. C. Aichelburg and R. U. Sexl, Gen. Rel. Grav. 2 (1971) 303

[15] J. B. Griffiths and J. Podolský, Phys. Lett. A 236 (1998) 8 